\title{
SUBALGEBRAS OF $B(X)$
}

\section{ALBERT WILANSKY}

Abstract. Two classes of subalgebras of the bounded operators on a Banach space $X$ are introduced. This gives an abstract setting, generalizations, and some shorter proofs of results in summability which are the special case $X=c$.

1. Introduction. With $X$ a nonreflexive Banach space we introduce two classes of subalgebras of $B(X)$, the set of all bounded operators on $X$. In $\$ 4$, we shall show that setting $X=c$, the space of convergent sequences of complex numbers, yields simplified proofs, and, in some cases, generalizations, of theorems of I. D. Berg, J. P. Crawford, R. J. Whitley, D. R. Kerr, H. H. Stratton and H. I. Brown. In $\$ 5$ we show that some of the results found in the special case $X=c$ continue to hold in the general setting. Our subalgebras have certain scalar homomorphisms defined on them in a natural way; these specialize, in the case $X=c$, to well known and important parameters used in summability theory.

Note. In all topological statements, $B(X)$ is to have the uniform norm, $\|T\|=\sup \{\|T x\|:\|x\| \leqq 1\}$.

2. Acknowledgment. The idea of using the adjoint in summability theory originated with J. P. Crawford and R. J. Whitley. In particular, many of the techniques used here follow naturally from ideas to be found in Crawford's Ph.D. Thesis. I have also had very helpful consultations with D. J. H. Garling.

3. The subalgebras. Let $w \in X^{\prime \prime} \backslash \hat{X}$. Here $X^{\prime \prime}$ is the second conjugate of $X$, and $\hat{X}$ is the image of $X$ under the natural embedding of $X$ into $X^{\prime \prime}$. Let $\Gamma_{w}^{a}=\left\{T \in B(X): T^{\prime \prime} w \in \hat{X} \oplus w\right\}$. Here $T^{\prime \prime}$ is the adjoint of the adjoint $T^{\prime}$ of $T$; and, for a vector subspace $S$ and vector $w, S \oplus w$ $=\{s+t w: s \in S, t$ scalar $\}$. For $T \in \Gamma_{w}^{a}, T^{\prime \prime} w=\hat{x}+\rho_{w}(T) w$, thus defining $\rho_{w}: \Gamma_{w}^{a} \rightarrow C$ where $C$ is the complex numbers. (The " $a$ " stands for "almost", see the definition below.)

We shall now hold $w$ fixed and drop the subscript. The dependence on $w$ is to be understood throughout.

Received by the editors September 24, 1970.

AMS 1970 subject classifications. Primary 46J10, 46J30, 47A05, 47B05; Secondary $40 \mathrm{C} 05,40 \mathrm{H} 05$.

Key words and phrases. Subalgebras, operators on Banach space, matrix algebras, summability. 
THeOREM 1. $\Gamma^{a}$ is a closed subalgebra of $B(X) ; \rho$ is a nonzero continuous scalar homomorphism on $\Gamma^{a}$.

For $S, \quad T \in \Gamma^{a}, \quad(S T)^{\prime \prime} w=S^{\prime \prime} T^{\prime \prime} w=S^{\prime \prime}[\hat{x}+\rho(T) w]=(S x)^{\wedge}+$ $\rho(T) \rho(S) w$. Thus $S T \in \Gamma^{a}$ and $\rho$ is multiplicative. Next, continuity of $\rho$ follows from the fact that its kernel, $\rho^{\perp}$, is closed; indeed it is the inverse image of $\hat{X}$ under the continuous map $T \rightarrow T^{\prime \prime} w$. Since $\rho$ is a continuous scalar homomorphism and $\rho(I)=1$ it follows that $\|\rho\|=1$. To see that $\Gamma^{a}$ is closed, let $T_{n} \rightarrow T$, say $T_{n}^{\prime \prime} w=\hat{x}_{n}+\rho\left(T_{n}\right) w$. Then $\left\|x_{m}-x_{n}\right\|=\left\|\hat{x}_{m}-\hat{x}_{n}\right\| \leqq 2\left\|T_{m}-T_{n}\right\| \cdot\|w\|$ so that $\left\{x_{n}\right\}$ is convergent, say to $x$. It follows that $\rho\left(T_{n}\right) w \rightarrow T^{\prime \prime} w-\hat{x}$, hence $T^{\prime \prime} w-\hat{x}$ is a multiple of $w$ so that $T \in \Gamma^{a}$.

The second subalgebra $\Gamma=\Gamma_{w}$ is the set of all $T \in B(X)$ for which $w$ is an eigenvector of $T^{\prime \prime}$. Clearly $\Gamma \subset \Gamma^{a}$ and for $T \in \Gamma, T^{\prime \prime} w=\rho(T) w$; that is, $\rho(T)$ is the corresponding eigenvalue. By Theorem $1, \rho$ is a continuous scalar homomorphism on $\Gamma$, and since $\rho(I)=1$, again $\|\rho\|=1$. It is obvious that $\Gamma$ is a closed subalgebra.

THEOREM 2. $\Gamma$ is a closed subalgebra of $\Gamma^{a} ; \rho$ is a nonzero continuous scalar homomorphism on $\Gamma$.

Definition. A member of $\Gamma$ will be called a w-matrix; a member of $\Gamma^{a}$ will be called an almost-w-matrix. An almost-w-matrix $T$ is called conull if $\rho(T)=0$, otherwise coregular.

This definition is justified on historical grounds by the specialization in $\$ 4$.

We now see that every $\Gamma_{w}^{a}$ includes all the weakly compact operators, hence all the compact operators.

THEOREM 3. Each weakly compact operator is a conull almost-wmatrix for all $w$.

This is merely a restatement of [3, Theorem 2, p. 482].

A compact operator need not be a $w$-matrix; for example, for $z \in X, f \in X^{\prime}$ let $z \otimes f$ be the one dimensional operator which carries $x$ to $f(x) z$. The formula $(z \otimes f)^{\prime \prime} w=w(f) \hat{z}$ reveals the following facts: $z \otimes f$ is an almost-w-matrix, and is a $w$-matrix if and only if $w(f)=0$.

4. Specialization. We now see how a classical sequence space theory is a special case of the above. Let $c$ be the Banach space of all convergent complex sequences and $T \in B(c)$. Defining $P_{n} \in c^{\prime}$ by $P_{n}(x)$ $=x_{n}$ we have $\left(P_{n} \circ T\right)(x)=(T x)_{n}=\chi_{n} \lim x+\sum a_{n k} x_{k}$. (See [5, $\S 6.4$, Example 5]. This reference covers some of the following remarks also.) Making the usual identification of $c^{\prime \prime}$ with $m$ (all bounded sequences) we have $T^{\prime \prime}: m \rightarrow m$ given by the matrix 


$$
\left(\begin{array}{cccc}
\chi & a_{1} & a_{2} & \ldots \\
\chi_{1} & a_{11} & a_{12} & \ldots \\
\chi_{2} & a_{21} & a_{22} & \ldots \\
\ldots & \ldots & \ldots
\end{array}\right) .
$$

The numbers in the first row are those which occur in the representation of $\lim \circ T \in c^{\prime}$ as $(\lim \circ T)(x)=\lim (T x)=\chi \lim x+\sum a_{k} x_{k}$.

Theorem 4. Let $T \in B(c)$. Then $T$ is a matrix if and only if $T$ is a $w$-matrix with $w=(1,0,0, \cdots) \in m=c^{\prime \prime}$. Moreover, with this choice of $w, \rho(T)=\chi$.

Clearly $T^{\prime \prime}(w)=\left(\chi, \chi_{1}, \chi_{2}, \cdots\right)$ is a multiple of $w$ (namely $\left.\chi \cdot w\right)$ if and only if $\chi_{1}=\chi_{2}=\cdots=0$. But this is precisely the condition that $T$ is given by the matrix $\left(a_{n k}\right)$.

REMARK 1. Theorems 2 and 4 yield a trivial proof of the classical fact that $\chi$ is a scalar homomorphism on the algebra of conservative matrices, that is, matrix members of $B(c)$. See, for example, [5, $\$ 14.1$, Problem 34].

REMARK 2. Theorem 3 now specializes to Steinhaus' classical result that a matrix summing all bounded sequences cannot be regular, i.e., cannot sum each convergent sequence to its ordinary limit.

We next turn to the almost matrices. An operator $T \in B(c)$ is called an almost matrix if $\left\{\chi_{n}\right\}$ is convergent. The space $\Gamma^{a}$ of almost matrices in $B(c)$ was shown in [2] to be the domain of the maximal extension, $\rho$, of $\chi$ as a scalar homomorphism.

THEOREM 5. Let $T \in B(c)$. Then $T$ is an almost matrix if and only if $T$ is an almost-w-matrix with $w=(1,0,0, \cdots) \in m \in c^{\prime \prime}$. Moreover, with this choice of $w, \rho(T)=\chi-\lim \chi_{n}$.

If $T$ is an almost matrix, $T^{\prime \prime} w=\left(\chi, \chi_{1}, \chi_{2}, \cdots\right)=$ $\left(\lim \chi_{n}, \chi_{1}, \chi_{2}, \cdots\right)+\left(\chi-\lim \chi_{n}\right) w \in \hat{c} \oplus w$. Conversely, if $T$ is an almost-w-matrix, $\left(\chi, \chi_{1}, \chi_{2}, \cdots\right)=T^{\prime \prime} w=\hat{x}+\rho w \in c$ and so $T$ is an almost matrix.

REMARK. Theorems 1 and 5 yield a trivial proof of the result [2, Theorem 3.4] that $\rho$ is a scalar homomorphism on the algebra of almost matrices in $B(c)$.

Note that the expression for $\rho$ in Theorem 5 is the same as that in Theorem $4 ; \chi$ is defined on $B(c)$ but is not a scalar homomorphism even on $\Gamma^{a}$.

5. Known results in the new setting. Throughout this section, $X, w, \Gamma$ and $\Gamma^{a}$ have the meanings given in $\$ 3$. A significant new di- 
rection in summability theory was taken by Berg [1] with the introduction of topological divisors of zero. See [6].

The following result extends the second part of Theorem 5 in [6].

Theorem 6. The following conditions are equivalent for $T \in B(X)$ :

(i) $T$ is a left topological divisor of 0 in $\Gamma$.

(ii) $T$ is a left topological divisor of 0 in $\Gamma^{a}$.

(iii) $T$ is a left topological divisor of 0 in $B(X)$.

(iv) $T$ is not a homeomorphism (into).

Here condition (i) means that for each $\epsilon>0$, there exists $s \in \Gamma$ with $\|S\|=1,\|T S\|<\epsilon$. That (iii) implies (iv) is Theorem 3.5 of [7]. It remains to prove that (iv) implies (i). Let $N=w^{\perp} \subset X^{\prime}$. It is proved in $[5, \S 11.2$, Problem 22] that $N$ is norming; this means that the norm on $X$ is equivalent to $\|\cdot\|_{N}$, where $\|x\|_{N}$ $=\|\hat{x} \mid N\|=\sup \{|f(x)|: f \in N,\|f\| \leqq 1\}$. In particular, $\|x\|_{N}>\delta$ for all $x$ with $\|x\|=1$, where $\delta$ is some fixed positive number. By (iv), for each $\epsilon>0$, there exists $x$ with $\|x\|=1,\|T x\|<\epsilon \delta$. By the norming condition, there exists $f \in w^{\perp}$ with $\|f\| \leqq 1 / \delta,|f(x)|=1$. Let $S=x \otimes f$ as defined in $\S 3$, where it is also pointed out that $S \in \Gamma$. Also $\|S\| \geqq 1$ since $\|S x\|=\|f(x) x\|=1=\|x\|$. Finally $\|T S\|<\epsilon$ for, given $y$ with $\|y\|=1$, we have $\|T S y\|=\|T[f(y) x]\|=|f y| \cdot\|T x\|<(1 / \delta) \cdot \epsilon \delta=\epsilon$.

The next two results extend and give simplified proofs for Theorems 1 and 2 of [6].

THeORem 7. The sets $\Gamma^{0}$ and $\Gamma^{a 0}$ of conull w-matrices and conull almost-w-matrices are left ideals in $B(X)$.

For $A \in \Gamma^{a 0}, T \in B(X)$ we have $(T A)^{\prime \prime} w=T^{\prime \prime} A^{\prime \prime} w=T^{\prime \prime} \hat{x}=(T x)^{\wedge}$. The other half of the proof is similar.

These left ideals need not be right ideals; see the first example on p. 250 of [6].

THEOREM 8. The subalgebras $\Gamma$ and $\Gamma^{a}$ of $B(X)$ are inverse-closed; indeed if $T \in \Gamma$ (or $\left.\Gamma^{a}\right)$ any left inverse of $T$ also belongs to $\Gamma$ (or $\Gamma^{a}$ ).

We give the proof for $\Gamma^{a}$ only. It is the special case of the following Lemma in which $S T=I$.

Lemma. Let $S, T \in B(X)$. If $T, S T$ are both almost-w-matrices with with at least one coregular, then $S$ is an almost-w-matrix.

First, $T$ must be coregular since otherwise, both $T$ and $S T$ would be conull by Theorem 7 . Let $\lambda=\rho(T), \mu=\rho(S T)$. Then 


$$
\begin{aligned}
S^{\prime \prime} w & =S^{\prime \prime}\left[(1 / \lambda) T^{\prime \prime} w-(1 / \lambda) \hat{x}\right]=(1 / \lambda)(S T)^{\prime \prime} w-(1 / \lambda)(S x)^{\wedge} \\
& =(1 / \lambda) \hat{y}+(1 / \lambda) \mu w-(1 / \lambda)(S x)^{\wedge} \in \hat{X} \oplus w .
\end{aligned}
$$

We finally generalize Corollary 3 , p. 247 of [6]. The result is given there for matrices; thus the generalization is new even for $X=c$ inasmuch as it extends to almost matrices. The following result can be carried much further, namely, we can prove that a conull almost$w$-matrix cannot have closed range and finite-dimensional, or even reflexive null space. The result given here merely shows that it cannot be a homeomorphism, that is, one to one and with closed range.

Theorem 9. A conull almost-w-matrix must be a left topological divisor of zero.

Suppose that $T$ is not a left topological divisor of zero, hence, Theorem 6, a homeomorphism. Since $T$ is conull we have $T^{\prime \prime} w=\hat{y}$ and we shall show that $y$ is in the range of $T$. Let $f \in X^{\prime}$ with $T^{\prime} f=0$. Then $f(y)=\hat{y}(f)=\left(T^{\prime \prime} w\right)(f)=w\left(T^{\prime} f\right)=0$. By [3, p. 479, Lemma 8], $y$ is in the range of $T$, say $y=T x$. For any $h \in X^{\prime}$ we have $h=T^{\prime} g$ for some $g \in X^{\prime}$ by $\left[3\right.$, p. 487, Theorem 2] and so $w(h)=w\left(T^{\prime} g\right)$ $=\left(T^{\prime \prime} w\right) g=\hat{y}(g)=g(y)=g(T x)=\left(T^{\prime} g\right) x=h(x)=\hat{x}(h)$. This leads to the conclusion $w=\hat{x}$ which contradicts the definition of $w$.

6. A generalization. Let $W$ be a closed proper subspace of $X^{\prime}$ which is norming (see the discussion in the proof of Theorem 6). Let $\Gamma_{W}$ be the set of all $T \in B(X)$ with the property that there exists $\rho=\rho(T)$ such that $\left(T^{\prime}-\rho \cdot I\right)\left[X^{\prime}\right] \subset W$. (Here $I$ is the identity map on $X^{\prime}$.) It is trivial that $\rho$ is uniquely defined and is linear. To see that $W$ is a subalgebra of $B(X)$ and that $\rho$ is a scalar homomorphism, let $S, T \in W, \rho(S)=\rho, \rho(T)=\lambda$. Then

$$
\begin{aligned}
{\left[(S T)^{\prime}-\lambda \rho I\right](f) } & =T^{\prime} S^{\prime} f-\lambda \rho f=T^{\prime}[w+\rho f]-\lambda \rho f \\
& =T^{\prime} w+\rho\left(w_{1}+\lambda f\right)-\lambda \rho f=T^{\prime} w+\rho w_{1} \\
& =w_{2}+\lambda w+\rho w_{1} \in W .
\end{aligned}
$$

Since also $\rho(I)=1, \rho$ is nonzero. Continuity of $\rho$ follows from the easily proved fact that its kernel is closed. To see that $\Gamma_{W}$ is closed, let $T_{n} \in \Gamma_{W}, T_{n} \rightarrow T$. Since $\|\rho\|=1,\left\{\rho\left(T_{n}\right)\right\}$ is convergent, say $\rho\left(T_{n}\right)$ $\rightarrow \lambda$. Then for all $f \in X^{\prime},\left[T_{n}^{\prime}-\rho\left(T_{n}\right) I\right](f) \in W$ and so, letting $n \rightarrow \infty$, $\left(T^{\prime}-\lambda I(f)\right) \in W$.

To see that the subalgebra $\Gamma_{w}$ is a special case, fix $w, X$ as in $\S 3$ and let $W=w^{\perp}$. Then $W$ is norming, as pointed out in the proof of Theorem 6; also $\Gamma_{w}=\Gamma_{W}$, for let $T \in \Gamma_{w}$. For $f \in X^{\prime}$, let 
$g=\left[T^{\prime}-\rho(T) I\right] f$. Then $w(g)=\left[T^{\prime \prime} w-\rho(T) w\right] f=0$ so that $g \in W$. Conversely, if $T \in \Gamma_{W}$, for all $f \in X^{\prime}$ we have

$$
\left(T^{\prime \prime} w-\rho w\right) f=w\left[\left(T^{\prime}-\rho I\right) f\right]=0
$$

since $\left[T^{\prime}-\rho I\right] f \in W$. Thus $T^{\prime \prime} w=\rho w$ and so $T \in \Gamma_{w}$.

In this more general setting we get the theorems of $\$ 5$. For example Theorem 6 used only the fact that $w^{\perp}$ is norming, hence the same proof works.

7. Coregular and conull. In [6] we used conull to mean $\chi=0$. By Theorem 4 , this agrees with our present usage for matrices. However, for almost matrices $\rho$ and $\chi$ are different (Theorem 5) and it is $\rho$ that is the more useful parameter. It is multiplicative and also plays a role in discussing maps of $c$ which $\chi$ does not. For example Theorem 3 of [6] says that no conull matrix map of $c$ can be a homeomorphism. It goes on to construct such a homeomorphism which is an almost matrix with $\chi=0$. But, we can add the result that there is no conull (meaning $\rho=0$ ) almost matrix map $T$ of $c$ which is a homeomorphism. To prove this we use Lemma 10 of [6] to construct a left inverse $S$ for $T$, contradicting Theorem 7 , above.

We also note that every projection of $c$ onto $c_{0}$ is a coregular almost matrix, for it has the form $I-u \otimes \lim$, where $u$ is an arbitrary member of $c$. (See [4, p. 547].)

\section{REFERENCES}

1. I. D. Berg, A Banach algebra criterion for Tauberian theorems, Proc. Amer. Math. Soc. 15 (1964), 648-652. MR 29 \#2574.

2. H. I. Brown, D. R. Kerr and H. H. Stratton, The structure of $B[c]$ and extensions of the concept of conull matrix, Proc. Amer. Math. Soc. 22 (1969), 7-14.

3. N. Dunford and J. T. Schwartz, Linear operators. I: General theory, Pure and Appl. Math., vol. 7, Interscience, New York, 1958. MR 22 \#8302.

4. A. E. Taylor, The extension of linear functionals, Duke Math. J. 5 (1939), 538-547. MR 1, 58.

5. A. Wilansky, Functional analysis, Blaisdell, New York, 1964. MR 30 \#25.

6. - Topological divisors of zero and Tauberian theorems, Trans. Amer. Math. Soc. 113 (1964), 240-251. MR 29 \#6222.

7. B. Yood. Transformations between Banach spaces in the uniform topology, Ann. of Math. (2) 50 (1949), 486-503. MR 10, 611.

Lehigh University, Bethlehem, Pennsylvania 18015 